\title{
CONSTRUTO PARA O CONCEITO DE WAYFINDING NA CIÊNCIA DA INFORMAÇÃO
}

\section{CONTRUCTION FOR THE CONCEPT OF WAYFINDING IN INFORMATION SCIENCE}

Arthur Ferreira Camposa

Fernando Luiz Vechiatob

\begin{abstract}
RESUMO
Introdução: Wayfinding está associado, no campo do Urbanismo, à orientação espacial dos sujeitos em espaços urbanos. Todavia, seus princípios podem ser aplicados em ambientes informacionais, visando facilitar a encontrabilidade da informação disponível, o que contribui significativamente para os estudos da Ciência da Informação. Objetivo: Apresentar os alicerces que delimitam o conceito de wayfinding na Ciência da Informação, conforme os estudos em Encontrabilidade da Informação. Metodologia: A partir de abordagem qualitativa e mediante a análise de conteúdo, são estudados artigos em âmbito nacional e internacional, encontrados no Portal de Periódicos da Capes e na Base de Dados em Ciência da Informação, considerando o período 2010 - 2020. A práxis foi percorrer o conteúdo desses artigos, compreendendo as categorizações e os núcleos de sentidos que fundamentam o conceito de wayfinding na Ciência da Informação. Resultados: É proposto um conceito de wayfinding convergente com este momento da história e cultura científicas. Conclusões: No momento histórico e cultural científico atual, as pesquisas sobre wayfinding têm potencial exponente na Ciência da Informação.
\end{abstract}

Descritores: Wayfinding. Encontrabilidade da Informação. Ambientes Informacionais. Informação e Tecnologia.

\section{INTRODUÇÃO}

Os sujeitos se empenham no processo de busca e uso da informação em um ambiente informacional a partir de necessidades plurais. Bibliotecas, Arquivos, Museus, Centros de Documentação, Centros de pesquisa, Web sites, Repositórios Digitais, Bibliotecas Digitais, Bases de Dados, fontes de

a Doutorando no Programa de Pós-Graduação em Ciência da Informação (PPGCI-UFPB). Email: arthurfcampos94@gmail.com.

b Doutor em Ciência da Informação pela Universidade Estadual Paulista (UNESP). Docente do Departamento de Ciência da Informação da Universidade Federal do Rio Grande do Norte (UFRN). E-mail: vechiato2008@Gmail.com 
informação em geral, são exemplos de ambientes informacionais analógicos, digitais e/ou híbridos.

No que remete a Informação e Tecnologia, a Ciência da Informação possibilita estudos em ambientes informacionais podendo otimizá-los, tornando autônomo o processo de Encontrabilidade da Informação (EI) pelos sujeitos. (VECHIATO; VIDOTTI, 2014). Para isso, um ambiente informacional deve ser planejado e estruturado mediante conceitos e construtos que levem em consideração a perspectiva dos sujeitos que utilizarão seus serviços e a informação propriamente dita.

Miranda (2019) compreende a Encontrabilidade mediante um mapeamento das propriedades do fenômeno informacional, ressaltando a Teoria da Intencionalidade. A autora destaca os estudos fenomenológicos e as propriedades da informação inserindo-os como um campo de estudos e pesquisa entre a Encontrabilidade e a Teoria da Intencionalidade.

A referida teoria está associada às características, experiências, comportamentos e habilidades dos sujeitos, o que corrobora a perspectiva da proposta de Vechiato e Vidotti (2014) ao delinearem um conceito de Encontrabilidade da Informação para a Ciência da Informação, posicionando-a como um processo informacional inserido nos ambientes informacionais.

A El subsidia planificações para a utilização da informação e seu impacto sobre o sujeito, trabalhando suas características em buscar, se orientar e acessar funcionalidades de um sistema ou ambiente informacional (VECHIATO; VIDOTTI, 2014). O wayfinding, um de seus atributos, se apresenta favorável para projetar mecanismos de sinalização de possíveis caminhos a serem seguidos pelo sujeito, de modo a favorecer sua orientação espacial.

Com o objetivo de apresentar os alicerces que delimitam o conceito de wayfinding na Ciência da Informação, utilizamos a análise de conteúdo para investigar como os autores em Ciência da Informação, no cenário nacional, e em Library and Information Science, no cenário internacional, trabalham o wayfinding em contexto teórico-prático.

Temos a justificativa de solidificar o wayfinding na Ciência da Informação 
mediante a subsídios que constroem o conceito a ser utilizado em estudos subsequentes. Utilizamos principalmente a Encontrabilidade da Informação como ponte que une o wayfinding à Ciência da Informação.

\section{PROCEDIMENTOS METODOLÓGICOS}

Estruturamos este artigo conforme uma pirâmide invertida, dialogando teoricamente com autores da Arquitetura e Urbanismo, do Design e da Ciência da Informação, propondo um consenso sobre o wayfinding em ambientes informacionais. Estudamos suas referências no contexto da legibilidade ambiental / urbana e da legibilidade informacional.

Sob caráter qualitativo, operamos a análise de conteúdo (BARDIN, 2011) para explorar artigos de periódicos que discutem o wayfinding tanto em nível teórico quanto prático (análises e planejamento de ambientes informacionais). Conduzimos a análise de conteúdo com pesquisas nacionais em Ciência da Informação e internacionais em Library and Information Science. As fontes de informação utilizadas foram o Portal de Periódicos Capes e a Base de Dados em Ciência da Informação (Brapci). Optamos por buscar artigos mediante o termo 'wayfinding' visando a abrangência de pesquisa.

Utilizando o Portal da Capes, pesquisamos por assunto o termo 'wayfinding', refinando para estudos dentro do período de 2010 - 2020. Incluímos os tópicos Humans, Wayfinding e Libraries e direcionamos para Library and Information Science. Encontramos, internacionalmente, 39 artigos de periódicos como resultados e nenhum nacional. Percorremos o conteúdo destes e encontramos categorizações e núcleos de sentido que contribuem para um conceito de wayfinding na Ciência da Informação. Dentre os 39 artigos encontrados, foram selecionados 23 que exploram o wayfinding como um estudo prático para projetos em bibliotecas, sendo estes os materiais que mais se aproximaram do escopo da Ciência da Informação, conforme proposta deste artigo.

Utilizando a Brapci, inserimos o termo 'wayfinding' no campo de busca, direcionamos para recuperação de pesquisas contendo esse termo no texto completo e definimos a busca para o período de 2010 - 2020. Encontramos 27 artigos. Também percorremos o conteúdo destes e formulamos categorizações 
e núcleo de sentidos que contribuem para um conceito de wayfinding na Ciência da Informação. Dos 27 artigos encontrados, foram selecionados 26 que abordam o wayfinding no âmbito teórico e prático favorecendo a análise de conteúdo a partir de núcleos de sentido. Neste corpus 20 artigos abordam 0 wayfinding no contexto da El.

A fase de coleta de materiais para análise terminou em 21 de junho de 2020. Não encontramos artigos que abordem wayfinding no ano de 2020 para 'Library and Information Science' no Portal de Periódicos da Capes e nem pesquisas contendo esse termo no 'Título, palavra-chave e resumo' na Brapci. Desse modo, destacamos que neste primeiro semestre de 2020 não há artigos que dialoguem e contribuam com este presente enquadramento conceitual nas fontes de informação utilizadas. O corpus, portanto, foi formado por 23 artigos da literatura internacional somados a 26 da literatura nacional, totalizando 49 artigos.

\section{WAYFINDING NO CONTEXTO DOS ESTUDOS EM ENCONTRABILIDADE DA INFORMAÇÃO}

A El contempla bases teóricas e epistemológicas relacionadas ao comportamento dos sujeitos e às funcionalidades dos sistemas e ambientes informacionais (VECHIATO, 2013). Os estudos e pesquisas sobre a El surgem devido ao ser humano buscar e se orientar por mecanismos ao seu redor, tanto na perspectiva informacional quanto na perspectiva urbana / ambiental. Morville (2005) compreende essa busca e orientação como findability.

Morville (2005) introduz findability exemplificando diretamente como o usuário/leitor encontrou o seu referido material (livro). Segundo o teórico, essa busca pôde ser feita com: palavras-chave, pelo advento da computação ubíqua, pelo wayfinding ou pela tomada de decisão mediante a descoberta de informações. Este autor define findability conforme demonstrado no Quadro 1:

Quadro 1 - Definição de findability

\begin{tabular}{|c|l|}
\hline $\mathbf{1}$ & A qualidade que um objeto possui para ser localizado ou recuperado; \\
\hline $\mathbf{2}$ & O grau no qual um determinado objeto pode ser fácil de ser descoberto ou recuperado; \\
\hline $\mathbf{3}$ & O grau em que um sistema ou ambiente suporta navegação e recuperação. \\
\hline
\end{tabular}

Fonte: (MORVILLE, 2005, p. 4, tradução nossa). 
Observamos que findability compreende a localização, a navegação, a recuperação e a descoberta de determinados objetos, direcionando para a qualidade que esse objeto possui para ser encontrado. De acordo com Morville (2005, p. 4, tradução nossa),

Findability é uma qualidade que pode ser medida ao nível tanto do objeto que se deseja encontrar quanto do sistema em questão. Podemos estudar os atributos de um objeto em particular que o tornam com mais ou menos possibilidades de ser recuperado. $O$ título de um documento. A cor de um colete salva-vidas. A presença de um aviso por meio de uma etiqueta RFID embutida. E podemos avaliar a proporção com que um sistema global suporta a capacidade das pessoas para encontrarem o seu caminho e, a partir disso, encontrarem o que precisam.

Morville (2005) cria o termo para designar a capacidade de buscar, encontrar e usar a informação em ambientes informacionais digitais, porém as recentes pesquisas sobre El incorporam análises em ambientes informacionais analógicos, digitais e híbridos, avançando para além da web e além do digital. Sua concepção está associada às preocupações com os sujeitos informacionais e seus comportamentos, habilidades e experiências na interação com esses ambientes.

Diante desse cenário, Miranda (2019) acrescenta que a Teoria da Intencionalidade insere singularidade a fenômenos, destacando a Fenomenologia e direcionando esses fenômenos para os problemas de informação, como a encontrabilidade da informação pelo sujeito. Compreendemos segundo Miranda (2019, p. 138) que "a Teoria da Intencionalidade justifica a informação enquanto fenômeno" e

o que a fenomenologia faz por meio de sua doutrina da Intencionalidade é superar o desvio cartesiano contra a publicidade da mente, os desvios das realidades das coisas, ou seja, é contra as alucinações ou imaginações que impedem um sujeito de chegar à realidade da manifestação das coisas (MIRANDA, 2019, p. 138).

Miranda (2019) considera que a Intencionalidade auxilia no projeto e análise dos instrumentos teóricos/técnicos coincidentes com a realidade social do sujeito, sendo um mecanismo relevante na otimização da busca pela informação, favorecendo a experiência do sujeito. Desse modo, Morville (2005) direciona findability ao âmbito web e para o wayfinding (orientação espacial) do 
ser humano em lugares distintos, o que nos faz perceber a importância da Intencionalidade nos estudos de El.

Vechiato e Vidotti (2014) conduzem os estudos em Encontrabilidade para o âmbito informacional, assim como Miranda (2010; 2019), trabalhando com o sujeito e suas características de buscar, se orientar e acessar funcionalidades de um sistema ou ambiente informacional. Campos, Sousa e Oliveira (2019) ressaltam a Encontrabilidade da Informação se relacionando e dialogando com a Arquitetura da Informação (Al). Os estudos em Al sinalizam subsídios e elementos para a estruturação, desenho, organização e disposição de informações para ambientes informacionais (CAMPOS; SOUSA; OLIVEIRA, 2019).

Rosenfeld, Morville e Arango (2015) definem a Arquitetura da Informação ilustrando conceitos em um quadro. Abreviando,

[...] a Arquitetura da Informação é o desenho estrutural de ambientes de informação compartilhados; a combinação de sistemas de organização, rotulagem, busca e navegação em websites e intranets; a arte e ciência de estruturar produtos de informação e experiências que permitam a encontrabilidade e; uma disciplina emergente, de prática focada em trazer princípios de design e arquitetura para o ambiente digital, contemplando as facetas conteúdo, contexto e usuário (CAMPOS; SOUSA; OLIVEIRA, 2019, p. 3).

Campos, Sousa e Oliveira $(2019$, p. 8) destacam que "[...] a Arquitetura da Informação tem preponderância sobre a Encontrabilidade da Informação, no sentido de fornecer elementos sistêmicos aos ambientes analógicos, digitais e híbridos de informação". Compreendemos que a El pode servir como orientação para o sujeito numa Arquitetura de Informação e a Al pode favorecer e solucionar possíveis problemas de Encontrabilidade da Informação.

Vechiato e Vidotti (2014) definem atributos que promovem a encontrabilidade da informação em ambientes informacionais, quais sejam: taxonomias navegacionais; instrumentos de controle terminológico; folksonomias; metadados; mediação dos informáticos; affordances; wayfinding; descoberta de informações; acessibilidade e usabilidade; mediação dos profissionais da informação; mediação dos sujeitos informacionais; intencionalidade; e mobilidade, convergência e ubiquidade.

Miranda e Vechiato (2017, p. 12) abordam wayfinding demonstrando que "[...] podemos observar como a orientação espacial e intuitiva contribuem para a 
encontrabilidade da informação em ambientes analógicos e digitais". O wayfinding no contexto da Encontrabilidade da Informação é um atributo favorável a orientação conforme a navegação do sujeito num ambiente informacional.

Os estudos sobre wayfinding ressaltam a possibilidade dos sujeitos se orientarem a partir de elementos auxiliadores na tomada de decisão e navegação em ambientes informacionais. Entendemos que em um ambiente informacional bem estruturado e adequado conforme 0 wayfinding, possivelmente o sujeito encontra informações de forma autônoma.

O wayfinding possui referências interdisciplinares, sendo estudado na área de Arquitetura e Urbanismo, na área do Design, como também na área da Ciência da Informação. O sujeito deve ser autônomo para a navegação e busca num ambiente informacional, mediante uma estruturação favorável a essa autonomia. Partindo dos estudos em Encontrabilidade da Informação, o wayfinding é um atributo potencializador para a orientação e legibilidade autônoma dos sujeitos informacionais a partir de sinalizações, pistas, placas ou qualquer outro elemento norteador em ambientes informacionais analógicos e digitais (VECHIATO, 2013).

\subsection{WAYFINDING: BASES INTERDISCIPLINARES PARA LEGIBILIDADE AMBIENTAL / URBANA}

Wayfinding, associado a orientação espacial, relaciona-se a possibilidades de orientação, localização e sinalização (RIBEIRO, 2009, p. 28). Morville (2005) demonstra que "wayfinding" dialoga com "findability" e Vechiato e Vidotti (2014) consideram essa reflexão no contexto da El.

O wayfinding nasce, sobretudo, na área de Arquitetura e Urbanismo, referindo-se a elementos de orientação em ambientes urbanos (mapas, números de rua ou sinais de direção). Lynch (1960) traz essa discussão o planejamento urbano como elemento de wayfinding (ruas, avenidas, residências). Na Ciência da Informação, observamos que o planejamento de ambientes informacionais favorece a orientação do sujeito. Podemos observar elementos de wayfinding para o planejamento urbano (Figuras 1 e 2) reforçando o estudo de Lynch (1960). 


\section{Figura 1 - Mapa Turístico da cidade do Natal}

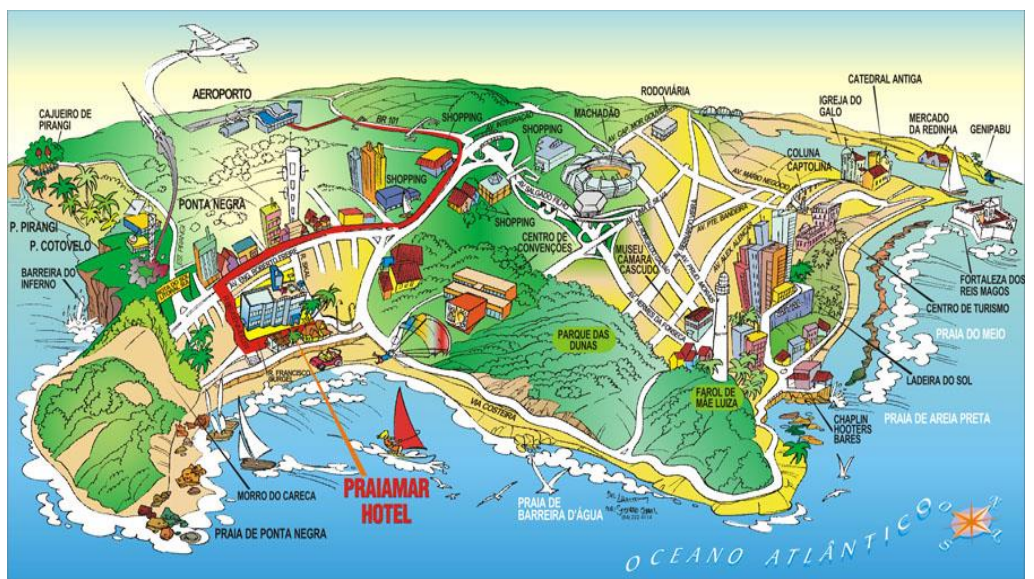

Fonte: III Simpósio Brasileiro de Componentes, Arquiteturas e Reutilização de Software (2009)

A Figura 1 indica um mapa turístico da cidade do Natal, no Estado do Rio Grande do Norte. Sua finalidade orienta espacialmente os membros participantes do III Simpósio Brasileiro de Componentes, Arquiteturas e Reutilização de Software (2009). Percebemos pontos conhecidos na cidade como a Praia de Ponte Negra, Morro do Careca e o Parque da Dunas, subsidiando a navegação e localização na cidade. É possível ter uma visão global da área em relação aos lugares (praias, aeroporto, caminhos para demais pontos turísticos etc.) e o Praiamar Hotel (sede do evento) é destacado em vermelho.

\section{Figura 2 - Linhas e sinalizações de Rua}

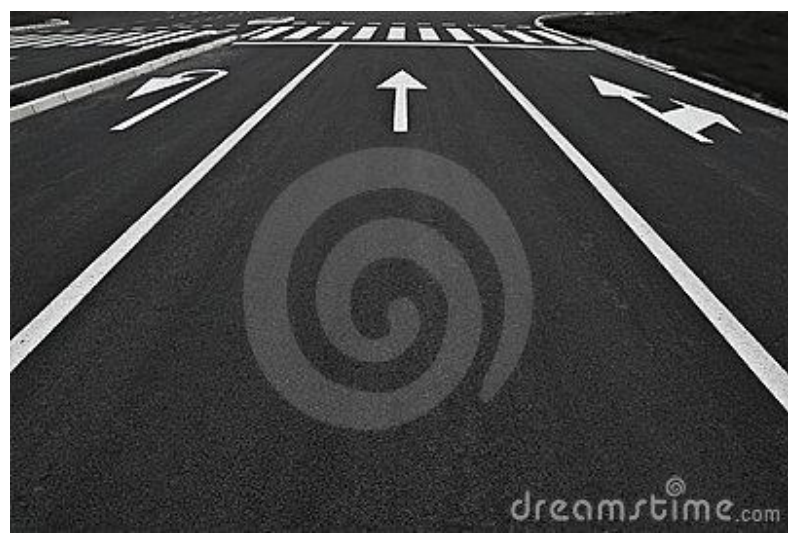

Fonte: extraído do site Dreamstime (2017)

A Figura 2 representa sinalizações presentes nas ruas das cidades, visando orientar o tráfego. Elas indicam caminhos que levam a determinados destinos. Numa biblioteca, por exemplo, são dispostos elementos e setas que representam caminhos e pistas possíveis para que o usuário siga. Lynch (1960) 
reforça a ideia de que o wayfinding é uma característica particular do sujeito

Estruturar e identificar o meio ambiente é uma atividade vital de todo o animal móvel. São muitas as espécies de orientações usadas: a sensação visual da cor, da forma, do movimento ou polaridade da luz, assim como outros sentidos, tais como o cheiro, o ouvido, o tacto, a cinestesia, a noção da gravidade, e talvez as de campos magnéticos ou elétricos. ( $\mathrm{LYNCH}, 1960, \mathrm{p}$. 13, tradução nossa).

Morville (2005) destaca que o wayfinding é instintivo nos seres humanos, sendo característico de cada sujeito. Relacionando a evolução humana, Morville (2005, p. 16, tradução nossa) indica que "nossos instintos de wayfinding testemunham o poder da evolução". Wayfinding contempla os atributos de direção humana num determinado ambiente e, como definição:

Wayfinding é uma palavra sofisticada para uma série de atividades que as pessoas conhecem e fazem para partir e chegar de um lugar para outro, dentro ou fora do determinado ambiente. Pode ser um impulso rápido ou oneroso, dependendo da pessoa, do meio ambiente em questão e da situação. Você pode pensar o wayfinding como um processo de cinco passos. Começa-se, primeiramente, em saber onde você está. Segundo, saber seu local de destino, para depois seguir a melhor rota para se chegar ao destino, ser capaz de reconhecer seu local de destino e encontrar o seu próprio caminho para chegar ao seu ponto de partida (CARPMAN; GRANT, 2002 apud MORVILLE, 2005, p. 17, tradução nossa).

Num ambiente informacional, os cinco passos de Carpman e Grant (2002) podem ser aplicados conforme orientação e localização autônoma. Ao buscarmos por uma informação, numa unidade de informação ou num web site, cognitivamente procuramos saber onde estamos, tomamos conhecimento do nosso local de destino para seguirmos a melhor rota, reconhecendo o local de destino e nosso ponto de partida com o intuito de percorrermos o caminho mais adequado para encontrarmos o que buscamos.

\subsection{WAYFINDING: BASES TEÓRICAS PARA LEGIBILIDADE EM AMBIENTES INFORMACIONAIS}

O wayfinding pode ser analisado quanto ao comportamento de usuários em ambientes informacionais digitais, na medida que um web site pode possuir pistas ou trilhas de navegação (breadcrumbs e landmarks) que ajudam na orientação e na navegação (MORVILLE, 2005). O autor indica que os estudos 
de wayfinding em ambientes informacionais da web se aproximam dos princípios de usabilidade na navegação.

A Figura 3 representa uma trilha de navegação (destacada em vermelho) no web site da Amazon Brasil, mapeada por uma busca pelos livros do detetive fictício Sherlock Holmes.

Figura 3 - Trilhas de navegação no site da Amazon Brasil em vermelho

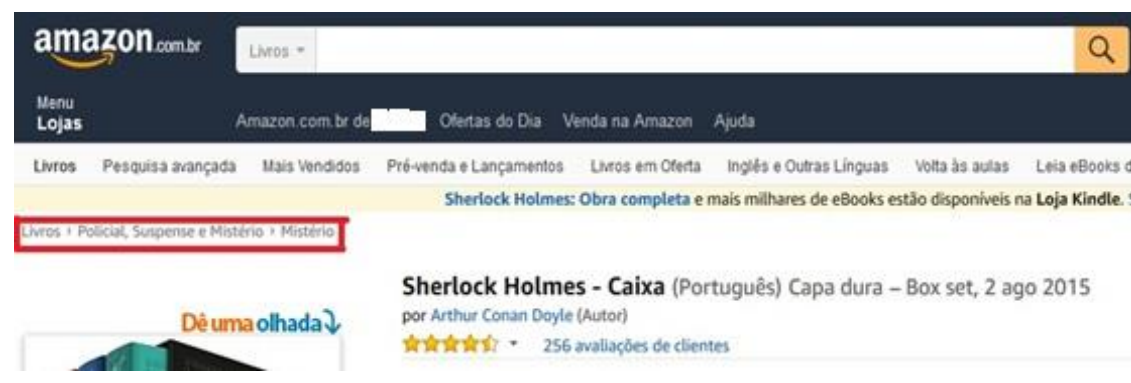

Fonte: extraído do site da Amazon Brasil (2017)

Lynch e Horton (2009) entendem o processo de wayfinding em quatro elementos cíclicos fundamentais, sendo eles: orientação (orientation), decisões de rota (route decisions), mapeamento mental (mental mapping), e encerramento (closure). A Figura 4 ilustra esses componentes.

Figura 4 - Elementos cíclicos do wayfinding

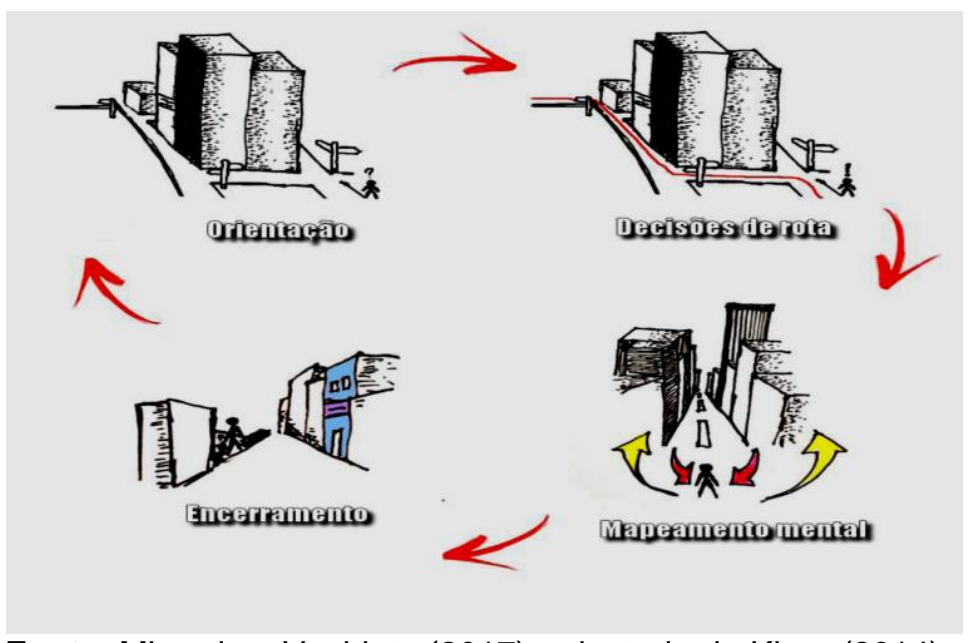

Fonte: Miranda e Vechiato (2017), adaptado de Khan (2014)

Lynch e Horton (2009) descrevem esse processo com perguntas: "Onde eu estou agora?", "Posso encontrar o caminho para onde quero ir?", "Minhas experiências são consistentes e compreensíveis o suficiente para saber onde estive e prever para onde devo ir em seguida?", "Posso reconhecer que cheguei ao lugar certo?" (MIRANDA; VECHIATO, 2017). 
Bins Elly (2002 apud MARTINS; ALMEIDA, 2014, p. 58) identifica que o wayfinding está ligado às formas com que um sujeito irá se localizar mentalmente ou se movimentar em um determinado ambiente. Cardoso et al. (2011, p. 11) enxergam que wayfinding é relacionado ao movimento orientado e "utiliza-se da aplicação dos recursos da sinalização para orientar e auxiliar os usuários a chegarem em determinado destino com segurança, tornando a experiência dos mesmos agradável".

De acordo com Smythe (2014, p. 21), "a orientação espacial é um processo natural que vivenciamos desde que nascemos". Esse processo se destaca como uma tarefa implícita no cognitivo do ser humano que o direciona, a partir de elementos presentes no espaço, a dirigir-se a um determinado caminho (SMYTHE, 2014).

Desse modo, o ambiente informacional orienta os usuários, a partir de estruturações sinalizadoras com a finalidade de se alcançar um caminho. Miranda e Vechiato $(2017$, p. 3) entendem que wayfinding

[...] é algo instintivo do ser humano, nós precisamos saber onde estamos, precisamos conhecer o caminho, devemos saber que rotas escolher e como nos localizar no espaço. A orientação espacial se dá por meio da interação do indivíduo com o meio em que se locomove.

Campos (2017) considera que wayfinding é um processo natural de todo ser humano, tornando-se uma característica particular, auxiliando os sujeitos na localização em ambientes (urbanos e informacionais analógicos, digitais ou híbridos). Um ambiente informacional pode conter elementos que colaboram na orientação e localização de espaço, assessorando a Encontrabilidade da Informação pelo sujeito.

A busca pela informação pode ser rápida ou devagar, pode conter obstáculos e desafios, pode ser prazerosa ou não prazerosa, ou pode-se chegar a um resultado esperado ou inesperado, contribuindo para a descoberta de informações. Essas variáveis dependem da percepção cognitiva dos sujeitos, tanto dos que buscam e acessam a informação, quanto dos que atuam na disponibilização e representação da informação. 


\title{
4 WAYFINDING NO CONTEXTO DOS ESTUDOS EM ENCONTRABILIDADE DA INFORMAÇÃO
}

\author{
Utilizando o Portal de Periódicos da Capes e a Base de Dados em
} Ciência da Informação (Brapci), observamos autores da Biblioteconomia e da Ciência da Informação a frente dessas pesquisas. Indicamos no Quadro 20 compilado de pesquisas encontradas no Portal da Capes. Nossa análise se baseia em referenciar o estudo encontrado (coluna esquerda do Quadro 2), realizando, como núcleo de sentido, um comentário sobre como o wayfinding é explorado na pesquisa destacada (coluna direita do Quadro 2). A organização está disposta em ordem ascendente conforme a data de publicação.

\section{Quadro 2 - Pesquisas sobre wayfinding no Portal Capes}

\section{REFERENCIA}

BISHOP, B. W.; MANDEL, L. H. Utilizing geographic information systems (GIS) in library research. Library hi tech, 2010.

LESNESKI, T. Big box libraries: beyond restocking the shelves with books. New library world, 2011.

HAHN, J. Location-based recommendation services in library book stacks. Reference Services Review, 2011.

KHOO, M.; ROZAKLIS, L.; HALL, C. A survey of the use of ethnographic methods in the study of libraries and library users. Library \& information science research, v. 34, n. 2, p. 82-91, 2012.

PELLEGRINO, C. Does telling them to ask for help work?: Investigating library help-seeking behaviors in college undergraduates.

Reference \& User Services Quarterly, v. 51 , n. 3, p. 272, 2012.

NORMORE, L. F. "Here be Dragons": A

Wayfinding Approach to Teaching Cataloguing. Cataloging \& classification quarterly, v. 50, n. 2-3, p. 172-188, 2012. HUMRICKHOUSE, L. New ADA rules take effect. American libraries, v. 43 , n. $5 / 6$, p. 24 25,2012

HAHN, J. Mobile augmented reality applications for library services. New library world, 2012.

\section{NÚCLEO DE SENTIDO}

A pesquisa busca sistemas de informação geográfica em bibliotecas como ferramenta para medir e analisar os serviços e estabelecer direções para os usuários. Wayfinding é trabalhado como uma tecnologia para direcionamento em sistemas.

Wayfinding é trabalhado como mecanismo para pistas visuais nos tetos das bibliotecas, sendo uma orientação intuitiva e inovadora para ambientes informacionais.

Wayfinding é analisado como mecanismo para divisão da biblioteca em regiões, com base em pesquisas validadas com usuários e recomendações de sistemas de informação.

Utiliza o wayfinding e o comportamento de busca de informações pelos usuários para analisar métodos etnográficos no estudo de usuários em bibliotecas.

Wayfinding é trabalhado como elemento para correção de problemas de sinalização em bibliotecas.

Wayfinding promove maneiras sobre como navegar a partir de recursos estruturais.

Wayfinding pode ser um mecanismo para criação de sinalizações que auxiliem indivíduos com deficiência visual, tornando essa sinalização 'amigável' com o usuário.

O Wayfinding em bibliotecas pode ser aumentado com a criação de um aplicativo de serviços que as bibliotecas oferecem. Um 
BISHOP, B. W. Analysis of reference transactions to inform library applications (apps). Library \& Information Science Research, v. 34, n. 4, p. 265-270, 2012. MANDEL, L. H. Finding their way: How public library users wayfind. Library \& Information Science Research, v. 35, n. 4, p. 264-271, 2013.

CHIA, Wai Ying. Mobile 3D Library Map: an innovative wayfinding tool for library users on the move. Library Hi Tech News, 2014.

AGARWAL, N. K.; LAWRENCE, H. Office location map of individuals in the library and other college campus buildings: A proof-ofconcept wayfinding system. Journal of Web Librarianship, v. 8, n. 3, p. 305-323, 2014. PAULUS, A. R. Using Data to Assess Staffing and Services: University of lowa Main Library. Journal of Access Services, v. 11, n. 3, p. 189-205, 2014.

GIVEN, L. M.; ARCHIBALD, H. Visual traffic sweeps (VTS): A research method for mapping user activities in the library space.

Library \& Information Science Research, v. 37, n. 2, p. 100-108, 2015.

WARREN, R.; EPP, C. Library Space and Signage Kindness Audits: What Does Your User See? Partnership: The Canadian Journal of Library and Information Practice and Research, v. 11, n. 1, 2016.

ANDREWS, C.; WRIGHT, S. E.; RASKIN, H. Library learning spaces: Investigating libraries and investing in student feedback. Journal of Library Administration, v. 56, n. 6, p. 647672, 2016.

ZAUGG, H.; CHILD, C. Collaborating with nonlibrary faculty for assessment and improved instruction. Journal of Library Administration, v. 56, n. 7, p. 823-844, 2016.

PORAT, L. User feedback as a management tool in academic libraries: a review.

Performance Measurement and Metrics, 2016.

KEISLING, B. L.; SPROLES, C. Reviewing and reforming library service points Lessons in review and planning services, building layout, and organizational culture. Library

Management, v. 38, n. 8-9, p. 426-436, 2017.

MANDEL, L. H. Understanding and describing users' wayfinding behavior in public library aplicativo dessa natureza pode otimizar a busca por livros nas estantes das bibliotecas.

Wayfinding é um atributo base para localização em bibliotecas e pode ser planejado a partir de perguntas pensadas pelos estudantes, como: 'Onde é a sala 105?'.

Deve-se aprofundar, como estratégia, o wayfinding em pesquisas que trabalhem com os processos cognitivos dos usuários de bibliotecas.

O wayfinding pode ser explorado como ferramenta na criação de um mapa 3D em bibliotecas, sendo uma ferramenta de orientação para seus funcionários e usuários.

O wayfinding torna-se um benefício para ferramentas de buscas interativas na web, sendo interativo também para grandes edifícios e organizações.

Wayfinding pode ser planejado e desenvolvido para bibliotecas universitárias a partir de perguntas direcionais cognitivas.

O wayfinding examina possibilidades com as quais os indivíduos ou clientes se orientam, navegam em espaços públicos e, com isso, pode ser explorado analisando como navegam os usuários de uma biblioteca pública. O foco seria na sinalização e na criação de marcadores físicos.

Importância do wayfinding intuitivo, orientação intuitiva em bibliotecas, já que projetos e designs complexos geram problemas de navegação, deixando difícil a orientação espacial pelo usuário com deficiência auditiva.

Nem todos os ambientes (bibliotecas) planejados agradam os estudantes e os bibliotecários otimizam esses ambientes por meio do wayfinding, pistas visuais, sinalizações, indicações que atendam às necessidades dos usuários.

Os autores citam e exemplificam um projeto de wayfindig (LibQUAL+®) que proporcionou amplas oportunidades para ensino de estudantes, reforçando que estudos sobre wayfinding em bibliotecas são relevantes.

Outra menção ao projeto LibQUAL+®B que gerou resultados sobre as dificuldades de estudantes ao localizarem materiais específicos na biblioteca da Universidade de Chicago

Os autores indicam problemas de sinalização e orientação devido a um projeto confuso de wayfinding em bibliotecas e serviços de bibliotecas.

Wayfinding sendo um estudo sobre como os seres humanos utilizam a variedade de mídia 


\begin{tabular}{|l|l|}
\hline $\begin{array}{l}\text { facilities. Journal of Librarianship and } \\
\text { Information Science, v. 50, n. 1, p. 23-33, } \\
2018 .\end{array}$ & $\begin{array}{l}\text { de informação para se orientarem e } \\
\text { navegarem em espaços, direcionando o } \\
\text { wayfinding para as bibliotecas públicas. }\end{array}$ \\
\hline $\begin{array}{l}\text { FERNANDEZ, P.; BRANNEN, M. "Through the } \\
\text { looking glass: envisioning new library } \\
\text { technologies" Media Production Part II: using } \\
\text { multimedia to tell stories. Library Hi Tech } \\
\text { News, 2018. }\end{array}$ & $\begin{array}{l}\text { Trata do uso de aplicações tecnológicas em } \\
\text { bibliotecas, permitindo buscas dinâmicas de } \\
\text { rotas a partir do wayfinding e informações } \\
\text { úteis para seus ambientes virtuais. }\end{array}$ \\
\hline $\begin{array}{l}\text { MANDEL, L. H.; JOHNSTON, M. P. Evaluating } \\
\text { library signage: A systematic method for } \\
\text { conducting a library signage inventory. } \\
\begin{array}{l}\text { Journal of Librarianship and Information } \\
\text { Science, v. 51, n. 1, p. 150-161, 2019. }\end{array}\end{array}$ & $\begin{array}{l}\text { Wayfinding é um auxiliar de sinalização, } \\
\text { dando suporte à navegação e fazendo parte } \\
\text { do processo de sinalização em bibliotecas. É } \\
\text { esperado que o wayfinding seja visto em } \\
\text { espaços públicos como as bibliotecas, sendo } \\
\text { um guia para a navegação. }\end{array}$ \\
\hline $\begin{array}{l}\text { CAFFREY, J.; SIMONE, J. Floor Map Images } \\
\text { and Accessibility: Providing Equivalent } \\
\text { Information with the User in Mind. Journal of } \\
\text { Web Librarianship, v. 13, n. 4, p. 283-295, 2019. }\end{array}$ & $\begin{array}{l}\text { O wayfinding pode guiar um mapa / caminho } \\
\text { de imagens e acessibilidade no chão para o } \\
\text { usuário se orientar, trabalhando e fornecendo } \\
\text { informações ao seu cognitivo. }\end{array}$ \\
\hline
\end{tabular}

Fonte: elaborado pelos autores (2020).

Dentre os 39 artigos encontrados, 23 exploram o wayfinding como um estudo prático para projetos em bibliotecas. Utilizamos esses 23 para a análise de conteúdo como critério de direcionamento para o diálogo com a Ciência da Informação. Ilustramos na Figura 5 as categorizações e núcleos de sentido investigados no Quadro 2.

Figura 5 - Categorizações e núcleos de sentido baseados em pesquisas no Portal Capes

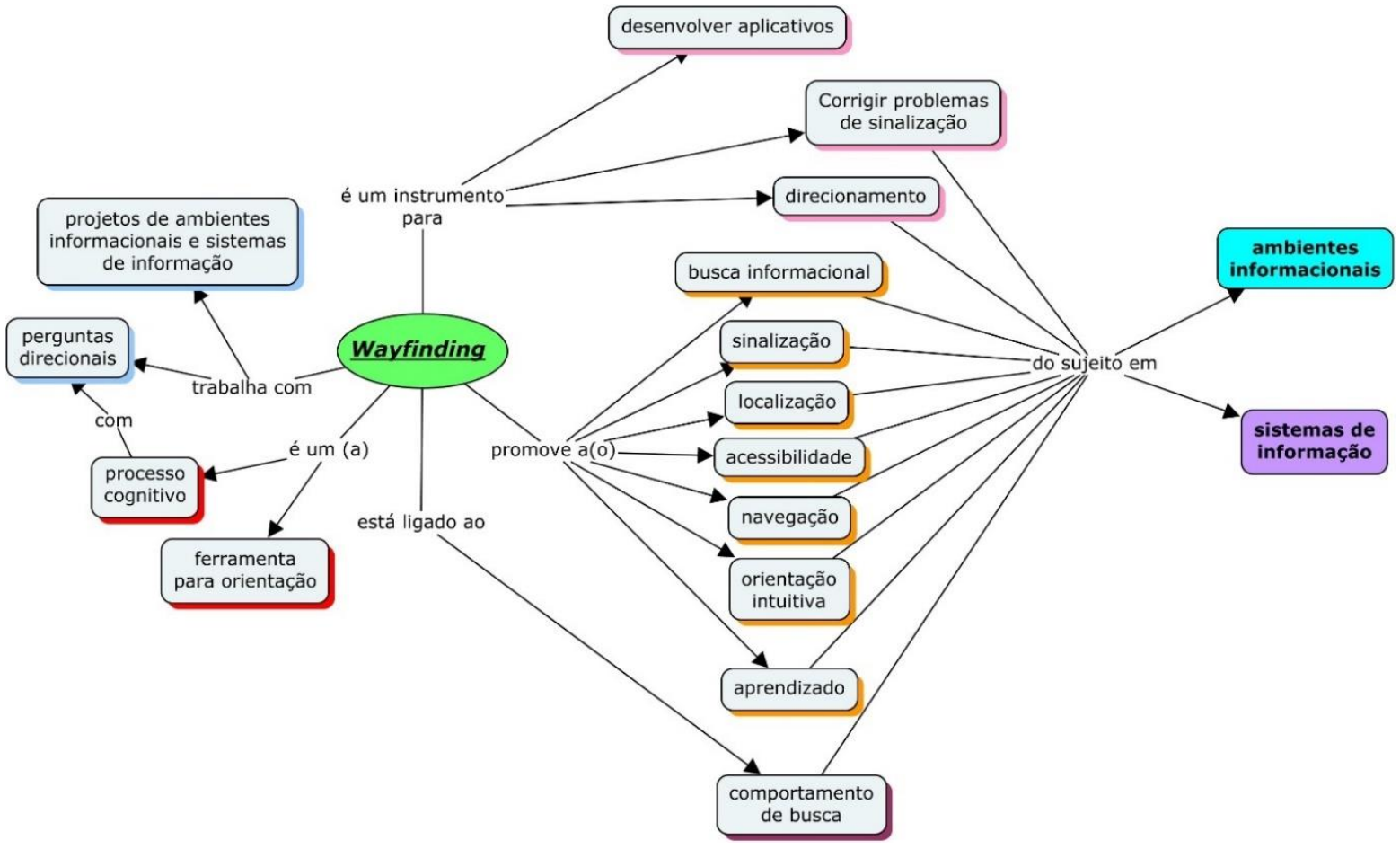

Fonte: elaborado pelos autores (2020) 
Analisando o mapa da Figura 5, o wayfinding promove a busca informacional, a sinalização, a localização, a acessibilidade, a navegação, a orientação intuitiva e o aprendizado do sujeito. É um instrumento que auxilia no desenvolvimento de aplicativos para dispositivos móveis, corrige problemas de sinalização e direciona o sujeito em ambientes informacionais e sistemas de informação. É uma ferramenta para orientação, sendo um processo cognitivo com perguntas direcionais; também está relacionado ao comportamento de busca do sujeito, a partir de projetos de ambientes informacionais e de sistemas de informação.

llustrando os resultados obtidos na Brapci mediante a busca descrita na seção de procedimentos metodológicos, o Quadro 3 demonstra pesquisas sobre o wayfinding encontradas. Da mesma forma supracitada, referenciamos o artigo encontrado do lado esquerdo do Quadro e o comentário sobre como o wayfinding é explorado na pesquisa do lado direito.

\section{Quadro 3 - Pesquisas sobre wayfinding na Brapci}

\begin{tabular}{|c|c|}
\hline REFERÊNCIA & NÚCLEO DE SENTIDO \\
\hline $\begin{array}{l}\text { VECHIATO, F. L.; VIDOTTI, S. A. B. G. } \\
\text { Encontrabilidade da Informação: atributos e } \\
\text { recomendações para ambientes } \\
\text { informacionais digitais. Informação \& } \\
\text { Tecnologia, p. 42-58, } 2014 \text {. }\end{array}$ & $\begin{array}{l}\text { Wayfinding é um atributo da Encontrabilidade } \\
\text { da Informação que, juntamente com as } \\
\text { affordances (pistas), auxiliam na navegação e } \\
\text { orientação espacial. Em relação a } \\
\text { complexidade, o wayfinding aplicado a web } \\
\text { torna-se limitado, porém com potencial } \\
\text { ecológico informacional para os estudos em } \\
\text { Arquitetura da Informação Pervasiva. }\end{array}$ \\
\hline $\begin{array}{l}\text { VECHIATO, F. L.; OLIVEIRA, H. P. C.; } \\
\text { VIDOTTI, S. A. B. G. Arquitetura da } \\
\text { informação Pervasiva e Encontrabilidade da } \\
\text { Informação: instrumento para a avaliação de } \\
\text { ambientes informacionais híbridos. } \\
\text { Informação \& Tecnologia, v. 3, n. 1, p. 47-65, } \\
2016 \text {. }\end{array}$ & $\begin{array}{l}\text { Associa o wayfinding a orientação espacial } \\
\text { mediante marcos e / ou metáforas que } \\
\text { auxiliem e dão pistas na localização, na } \\
\text { encontrabilidade e na descoberta de } \\
\text { informações por meio da navegação de } \\
\text { sujeitos em ambientes informacionais } \\
\text { híbridos. É indicado como um atributo da } \\
\text { Encontrabilidade da Informação. }\end{array}$ \\
\hline $\begin{array}{l}\text { PADUA, M. C.; DIAS, G. A. Desafios da } \\
\text { Arquitetura da Informação Pervasiva: } \\
\text { reduzindo a desorientação, aumentando a } \\
\text { legibilidade e wayfinding. Informação \& } \\
\text { Sociedade, v. } 26, \text { n. } 2,2016 \text {. }\end{array}$ & $\begin{array}{l}\text { Associa o wayfinding como um auxiliador no } \\
\text { desenvolvimento de processos e } \\
\text { metodologias em ambientes informacionais, } \\
\text { podendo maximizar a experiência do usuário } \\
\text { (UX) e reduzir a desorientação, tendo a } \\
\text { Arquitetura da Informação Pervasiva } \\
\text { (RESMINI; ROSATI, 2011) como um recurso. }\end{array}$ \\
\hline $\begin{array}{l}\text { FERREIRA, A. M. J. F. C.; VIDOTTI, S. A. B. } \\
\text { G. A Encontrabilidade da Informação em wel } \\
\text { sites de museus. Informação@Profissões, } \\
5, \text { n. 2, p. 79-101, } 2016\end{array}$ & $\begin{array}{l}\text { Wayfinding é um atributo da Encontrabilidade } \\
\text { da Informação e oferece subsídios para } \\
\text { orientar a navegação dos sujeitos em } \\
\text { ambientes informacionais digitais. }\end{array}$ \\
\hline $\begin{array}{l}\text { MIRANDA, E. B. S.; VECHIATO, F. L. } \\
\text { Wayfinding em ambientes informacionais: }\end{array}$ & $\begin{array}{l}\text { Wayfinding é um atributo da Encontrabilidade } \\
\text { da Informação, possibilitando a avaliação de }\end{array}$ \\
\hline
\end{tabular}


proposta de recomendações para avaliação no contexto da Encontrabilidade da Informação.

Revista Informação na Sociedade

Contemporânea, v. 1 n. 3, n. 3, p. 1-21, 2017. CAMPOS, A. F.; VECHIATO, F. L. Wayfinding no contexto da Encontrabilidade da Informação: avaliação dos ambientes informacionais do Departamento Estadual de Imprensa do Rio Grande do Norte.

Biblionline, v. 13, n. 2, p. 44-55, 2017.

FERNANDES, W. M.; VECHIATO, F. L.

Encontrabilidade da Informação no Repositório Institucional da Unesp: uma avaliação com dispositivos móveis. Encontro Nacional de Pesquisa em Ciência da Informação, n. XVIII ENANCIB, 2017.

VIDOTTI, S. A. B. G.; ROA-MARTÍNEZ, S. M.; CONEGLIAN, C. S.; FERREIRA, A. M. J. F. C.; VECHIATO, F. L. As contribuições das heurísticas de usabilidade para a

Encontrabilidade da Informação. Encontro

Nacional de Pesquisa em Ciência da

Informação, n. XVIII ENANCIB, 2017.

CUSTÓDIO, N. C.; VECHIATO, F. L.

Encontrabilidade da Informação em

Repositórios Institucionais: uma proposta de instrumento de avaliação. Revista

Informação na Sociedade Contemporânea,

n. Especial, p. 1-17, 2017.

SILVA, N. B. P.; VECHIATO, F. L.; VIDOTTI, S. A. B. G. Encontrabilidade da Informação no ambiente Technology, Entertainment and Design. Informação \& Informação, v. 22, n. 2, p. 481-496, 2017

MARQUES, C. A. G.; VECHIATO, F. L. Arquitetura da Informação em Repositórios Digitais: análise do Repositório Institucional da Universidade Federal do Rio Grande do Norte. Bibliocanto, v. 3 n. 1, n. 1, p. 2-28, 2017. SANCHEZ, F. A.; VECHIATO, F. L.

Encontrabilidade da informação em repositórios digitais: um enfoque nos repositórios institucionais da USP, Unesp e Unicamp. Encontro Nacional de Pesquisa em Ciência da Informação, n. XVIII ENANCIB, 2017.

NASCIMENTO, J. A.; CARVALHO, N. M. G. Encontrabilidade da informação e videoativismo: uma análise do atributo folksonomia. Encontro Nacional de Pesquisa em Ciência da Informação, n. XVIII ENANCIB, 2017.

NASCIMENTO, J. A.; CARVALHO, N. M. G. Encontrabilidade da informação e videoativismo: uma análise do atributo folksonomia no youtube. orientação em ambientes informacionais analógicos e digitais. Os autores criam diretrizes de recomendação de wayfinding para esses ambientes.

Wayfinding é um atributo da Encontrabilidade da Informação e é utilizado como instrumento de análise num ambiente informacional analógico e num ambiente informacional digital, a partir das diretrizes de recomendações propostas por Miranda e Vechiato (2017).

Wayfinding é um atributo da Encontrabilidade da Informação e é utilizado para avaliação de um ambiente informacional digital acessado em dispositivos móveis, explorando principalmente os breadcrumbs ou trilhas de navegação.

A usabilidade contempla o Wayfinding no contexto da Encontrabilidade da Informação.

Wayfinding é um atributo da Encontrabilidade da Informação e é utilizado como subsídio para um instrumento de avaliação em Repositórios Institucionais.

Wayfinding é um atributo da Encontrabilidade da Informação sendo um facilitador de localização de informações na interface de um ambiente informacional digital.

Wayfinding é um atributo da Encontrabilidade da Informação, associado a Arquitetura da Informação, sendo representado pelas trilhas de navegação no Repositório Institucional em questão.

Wayfinding é um atributo da Encontrabilidade da Informação mediante as trilhas de navegação disponíveis nos Repositórios estudados e a autonomia na navegação pelo sujeito.

Wayfinding é abordado como um atributo da Encontrabilidade da Informação.

Wayfinding é abordado como um atributo da Encontrabilidade da Informação. 


\begin{abstract}
Informação@Profissões, v. 6, n. 2, p. 60-76, 2017.

TANUS, G. F. S. C.; OLIVEIRA, E. D.; PAULA, M. G. Concepções de uma biblioteca do século XXI: protagonismo da Biblioteca Professora Etelvina Lima (ECI-UFMG). Revista Brasileira de Biblioteconomia e Documentação, v. 13, p. 1712-1730, 2017. OLIVEIRA, J. A. D. B. E.; JORENTE, M. J. V. Proposta de disciplina Design da Informação para Ciência da Informação. Encontro Nacional de Pesquisa em Ciência da

Destaca o wayfinding como um sinalizador especial de direção, juntamente com um caminho tátil garantindo a acessibilidade de usuários com deficiência visual na Biblioteca analisada.

Discorre sobre a disciplina de Design da Informação na Ciência da Informação, trabalhando o wayfinding como um sistema de sinalização.
\end{abstract} Informação, n. XVIII ENANCIB, 2017. BRANDT, M. B.; VECHIATO, F. L.; VIDOTTI, S. A. B. G. Encontrabilidade da informação na câmara dos deputados. Em Questão, v. 24, n. 1, p. 41-64, 2018

BRITO, J. F.; MATIAS, M. O Museu da Diversidade Sexual sob a ótica da Encontrabilidade da Informação. Encontro Nacional de Pesquisa em Ciência da Informação, n. XIX ENANCIB, 2018. REIS, S. G. O.; VECHIATO, F. L.; SANCHES, P. R.; CRUZ, D. G. Avaliação da Encontrabilidade da Informação do Repositório Institucional da Universidade Tecnológica Federal do Paraná. InCID: Revista de Ciência da Informação e Documentação, v. 9 n. 2, n. 2, p. 169-188, 2018.

FERREIRA, R. R.; ROCHA, L. M. G. M. Usabilidade da interface virtual de museus constituídos no plano físico. Encontro Nacional de Pesquisa em Ciência da Informação, n. XIX ENANCIB, 2018. ARAÚJO, A. K. S.; MAIA, F. H.; VECHIATO, F. L. Encontrabilidade da Informação em Repositórios Digitais. Revista Informação na Sociedade Contemporânea, v. 2 n. 1, n. 1, p. 1-15, 2018

ALVAREZ, E. B.; VIDOTTI, S. A. B. G.; SANCHEZ, J. A. P. Modelo para avaliar a Encontrabilidade da Informação em ambientes informacionais que usam Sistemas de Recomendações. Encontro Nacional de Pesquisa em Ciência da Informação, n. XIX ENANCIB, 2018.

SANCHEZ, F. A.; VECHIATO, F. L.

Encontrabilidade da Informação em Repositórios Institucionais: um estudo com eye-tracking. Encontro Nacional de Pesquisa em Ciência da Informação, n. XIX ENANCIB, 2018.

OLIVEIRA, J. A. D. B. E.; JORENTE, M. J. V. Design da informação e sua relevância para a ciência da informação. Encontros Bibli: Revista Eletrônica de Biblioteconomia e Ciência da Informação, v. 24, n. 54, p. 25-37,

Wayfinding é um atributo da Encontrabilidade da Informação e utilizado para avaliar uma ecologia informacional complexa.

Wayfinding é um atributo da Encontrabilidade da Informação sendo utilizado como instrumento de avaliação para ecologias informacionais complexas.

Wayfinding é um atributo da Encontrabilidade da Informação sendo utilizado para análise de um Repositório Institucional. Os autores o estudam no contexto da interface do ambiente informacional digital.

Wayfinding é um mecanismo que estrutura a navegação no âmbito da usabilidade na interface, convergindo com um processo cognitivo.

Wayfinding é um atributo da Encontrabilidade da Informação relacionado a navegação na interface do Repositório Digital analisado.

Wayfinding é um atributo da Encontrabilidade da Informação que possibilita a avaliação em ambientes informacionais que utilizam Sistemas de Recomendações.

Wayfinding é um atributo da Encontrabilidade da Informação para Repositórios Institucionais.

Aborda o wayfinding como um sistema de sinalização no contexto do Design da Informação. 


\begin{tabular}{|c|c|}
\hline & \\
\hline $\begin{array}{l}\text { INCHEZ, F. A.; VECHIATO, F. L.; VIDOTTI, } \\
\text { A. B. G. Encontrabilidade da informação em } \\
\text { positórios de Dados: uma análise do } \\
\text { taOne. Informação \& Informação, v. } 24, \text { n. }\end{array}$ & $\begin{array}{l}\text { Wayfinding é um atributo da Encontrabilidade } \\
\text { da Informação, auxiliando o sujeito } \\
\text { informacional com pistas e orientação } \\
\text { espacial ao navegar no Repositório de Dados } \\
\text { utilizado. Está ligado a descoberta de } \\
\text { informações por navegação. }\end{array}$ \\
\hline . & $\begin{array}{l}\text { Wayfinding é um atributo da Encontrabilidade } \\
\text { da Informação inserido no Sistema de } \\
\text { navegação da Arquitetura da Informação, } \\
\text { propiciando a orientação espacial na interface } \\
\text { de ambientes informacionais digitais. }\end{array}$ \\
\hline
\end{tabular}

Fonte: elaborado pelos autores (2020).

Dos 27 artigos encontrados, 26 abordam o wayfinding no âmbito teóricoprático favorecendo a investigação de categorizações a partir de núcleos de sentido. Em relação a Encontrabilidade da Informação, 20 artigos desses 26 destacados fazem alusão ao wayfinding nesse contexto. Direcionamos então a análise para os 26 como critério de especificidade para o construto do conceito de wayfinding na Ciência da Informação. A Figura 6 ilustra as categorizações e núcleos de sentido investigados no Quadro 3.

Figura 6 - Categorizações e núcleos de sentido baseados em pesquisas na Brapci

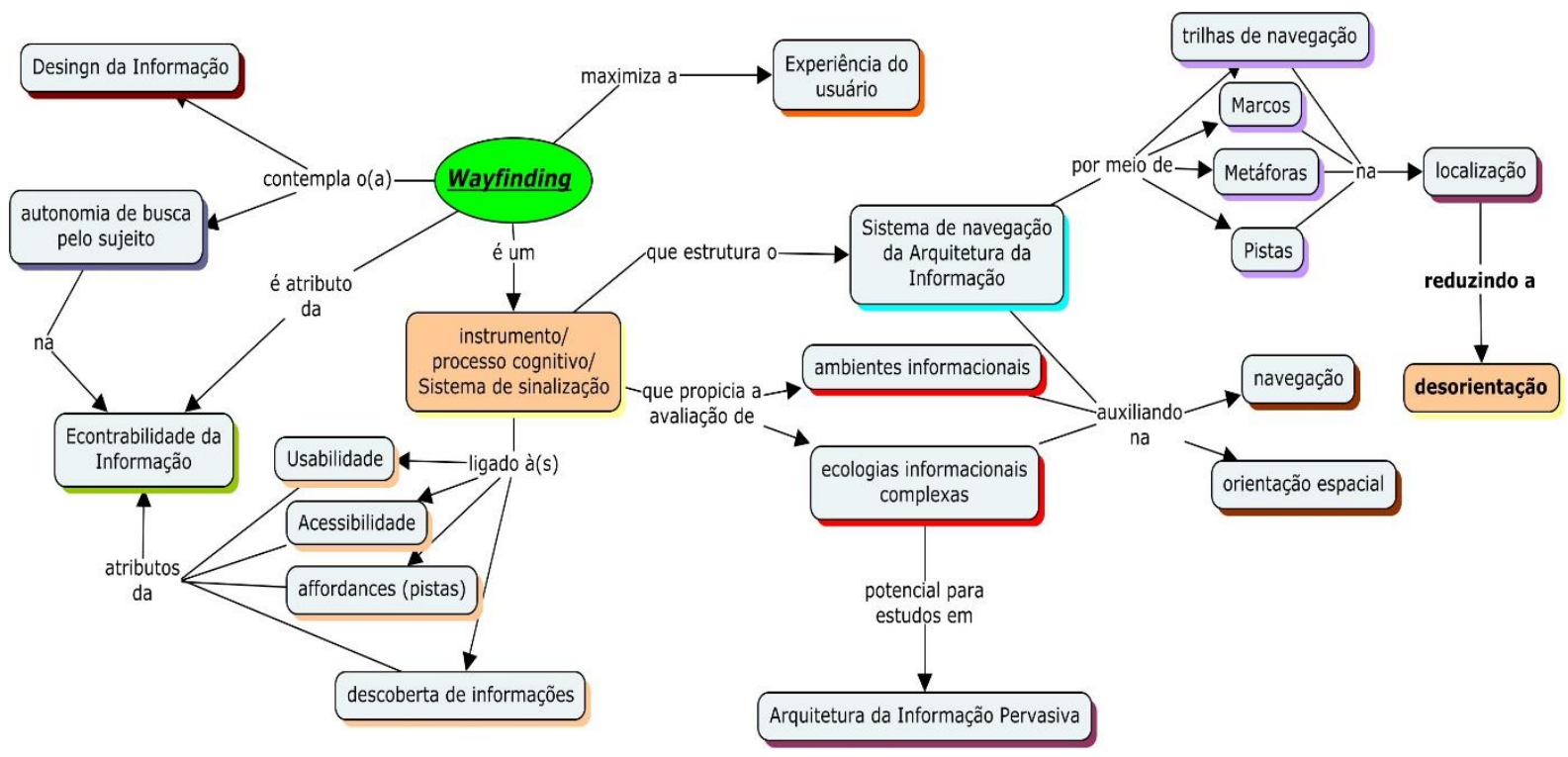

Fonte: elaborado pelos autores (2020)

Compreendemos o mapa da Figura 6 constatando que o wayfinding maximiza a experiência do usuário, sendo um instrumento ou processo 
cognitivo ou sistema de sinalização ligado à usabilidade, à acessibilidade, às affordances e à descoberta de informações, todos atributos da Encontrabilidade da Informação. Contempla a autonomia de busca pelo sujeito, contribuindo para estudos em Design da Informação. Propicia a avaliação de ambientes informacionais e ecologias informacionais complexas ${ }^{1}$, tendo sob essa última potencial para estudos em Arquitetura da Informação Pervasiva, auxiliando na navegação e na orientação espacial. $O$ wayfinding estrutura o Sistema de navegação da Arquitetura da Informação por meio de trilhas de navegação, marcos, metáforas e pistas, auxiliando na localização do sujeito e reduzindo a desorientação.

As ecologias informacionais complexas são o objeto de estudo e aplicação da Arquitetura da Informação Pervasiva. Esta é uma abordagem da Arquitetura da Informação que vai além dos ambientes web, compreendendo experiências holísticas ${ }^{2}$ e cross-channe $^{3}$ dos sujeitos. A Arquitetura da Informação Pervasiva entende os canais de informação interligados numa ecologia informacional complexa mediante a complexidade dos processos infocomunicacionais na sociedade pós-moderna, ou seja, um sujeito pode acessar o mesmo conteúdo informacional em diversos e distintos dispositivos e ambientes com o mesmo fim. Um exemplo desse tipo de experiência é iniciar uma compra num web site e-commerce e retirar o produto na loja, isto é, uma experiência que inicia no ambiente digital e termina no ambiente analógico. (OLIVEIRA; VIDOTTI; BENTES PINTO, 2015).

Diante dos resultados apresentados e discutidos, é apresentada na próxima seção uma proposta de conceito de wayfinding para o campo da Ciência da Informação.

1 "[...] conjunto de espaços, ambientes, canais, mídias, tecnologias e sujeitos com seus comportamentos, todos interligados e conectados de maneira holística pela informação" (OLIVEIRA, 2014, p. 134)

$2 \mathrm{O}$ todo e cada uma das partes interagem constantemente e de diversas maneiras.

${ }^{3}$ Interação e comunicação entre dois ou mais canais de informação. 


\section{PROPOSTA DE UM CONCEITO DE WAYFINDING PARA A CIÊNCIA DA INFORMAÇÃO}

Diante da leitura, análise de convergências e divergências, questionamentos, inferências, descrição nos Quadros 2 e 3 e por fim as categorizações que resultaram nos mapas conceituais dispostos nas Figuras 7 e 8, compreendemos como o wayfinding está sendo abordado nas pesquisas do período de 2010 - 2020. Observamos que o wayfinding é utilizado como um mecanismo precursor para que o sujeito se oriente e encontre informações em ambientes informacionais.

Nesse intervalo temporal, visualizamos que as pesquisas sobre wayfinding avançam com estudos que abarcam mais o digital do que 0 analógico. Pesquisas sobre o wayfinding em bibliotecas são exploradas em âmbito internacional, porém caminhando para investigações e projetos de ambientes informacionais digitais.

Em âmbito nacional, constatamos que os estudos em wayfinding estão, em maioria, atrelados aos estudos em Encontrabilidade da Informação. Dessa forma, percebemos que no presente momento o wayfinding é um subsidio para análises e projetos de ambientes informacionais que procuram dar autonomia de busca e orientação para o sujeito.

Portanto, compreendemos o Wayfinding, em relação aos seus pressupostos teóricos, como um estudo associado à Encontrabilidade da Informação e, consequentemente, passível de ser incorporado à Ciência da Informação. Em sua dimensão prática:

- é um instrumento de sinalização que auxilia no processo cognitivo do sujeito, maximizando suas experiências em sistemas de informação, em ambientes informacionais e em ecologias informacionais complexas;

- é um atributo da Encontrabilidade da Informação ligado a outros atributos como a usabilidade, a acessibilidade, as affordances e a descoberta de informações, promovendo a autonomia de busca pelo sujeito; 
- é uma característica instintiva do ser humano possibilitando estruturações no sistema de navegação da Arquitetura da Informação por meio de trilhas de navegação, marcos, metáforas e pistas em distintos ambientes informacionais;

- é uma ferramenta de direcionamento, de aprendizado, intencional no comportamento de busca do sujeito e útil para análise e projetos de sistemas de informação, ambientes informacionais e ecologias informacionais complexas.

\section{CONSIDERAÇÕES FINAIS}

Atingimos nosso objetivo conforme o delineamento do que foi proposto nos procedimentos metodológicos. Trazemos o conceito de wayfinding na Ciência da Informação propiciando um construto para pesquisas futuras. Observamos que, no momento histórico e cultural científico atual, as pesquisas sobre wayfinding tem potencial significativo na Ciência da Informação.

Sugerimos que conforme nossa consolidação do conceito disposto neste artigo, as análises em sistemas de informação, ambientes informacionais e ecologias informacionais complexas se direcionem também para dispositivos móveis, levando em consideração a responsividade, isto é, a adaptação de interfaces digitais nesses dispositivos.

Investigar o wayfinding em bibliotecas, arquivos e demais unidades de informação é uma necessidade também, visto que a orientação espacial é essencial para que o sujeito busque uma informação de maneira autônoma. Observamos que esses estudos são abordados em âmbito internacional e possibilitam, portanto, análises teórico-práticas em âmbito nacional.

É interessante um estudo que investigue as influências que o wayfinding exerce na descoberta de informações em Repositórios Digitais, Arquivos e Bibliotecas Digitais, bem como em web sites de e-commerce, jornalísticos e informacionais. Observamos também a necessidade de estudos que posicionem o wayfinding frente a web, web semântica e web pragmática. No contexto da Arquitetura da Informação, tomamos o conhecimento de que 
existem pesquisas com essa finalidade e, como o wayfinding promove estruturações no sistema de navegação e auxilia no sistema de busca.

\section{REFERÊNCIAS}

BARDIN, Laurence. Análise de conteúdo. Lisboa: Edições 70, 2011.

CAMPOS, Arthur Ferreira. Wayfinding no contexto da encontrabilidade da informação: uma análise dos ambientes informacionais do Centro de Documentação Núcleo Temático da Seca e do Semiárido da Universidade Federal do Rio Grande do Norte (NUT-SECA UFRN). 2017. 103f. Trabalho de Conclusão de Curso (Graduação em Biblioteconomia) - Universidade Federal do Rio Grande do Norte, Natal, 2017. Disponível em:

https://monografias.ufrn.br/jspui/handle/123456789/5687. Acesso em: 02 fev. 2020.

CAMPOS, Arthur Ferreira; SOUSA, Marckson Roberto Ferreira de; OLIVEIRA, Henry Poncio Cruz de. Relações teóricas possíveis entre Encontrabilidade da Informação e Arquitetura da Informação. ENCONTRO NACIONAL DE PESQUISA EM CIÊNCIA DA INFORMAÇÃO (ENANCIB), 20., 2019. Anais [...] Florianópolis: ANCIB; UFSC, 2019. Disponível em: http://hdl.handle.net/20.500.11959/brapci/122794. Acesso em: 21 jun. 2020.

CARDOSO, Eduardo et al. Contribuição metodológica em design de sinalização. InfoDesign: Revista Brasileira de Design da Informação, v. 8, n. 1, p. 10-30, 2011. Disponível em: https://infodesign.emnuvens.com.br/infodesign/article/view/107. Acesso em: 02 fev. 2020.

CARPMAN, Janet R.; GRANT, Myron A. Wayfinding: abroad view. In: BECHTEL; R. B.; CHURCHMAN, A. (ed.). Handbook of environmental psychology. New York: John Wiley, 2002. p. 427-443.

KHAN, Louis. The image of the city: Kevin Lynch. Qatar University. 2014.

LYNCH, Kevin. The image of the city. São Paulo: Massachusets Institute of Technology and the President and Fellowsof Harvard College, 1960.

LYNCH, Patrick James; HORTON, Sarah. Web style guide: basic design principles for creating web sites. 3. ed. 2009. Disponível em: http://webstyleguide.com/wsg3/. Acesso em: 02 fev. 2020.

MARTINS, Laura Bezerra; ALMEIDA, Maria de Fátima Xavier do Monte. O conceito de wayfinding na concepção de projetos arquitetônicos: Interdisciplinaridade a serviço da inclusão. ARCHITECTON-Revista de Arquitetura e Urbanismo, v. 4, n. 6, 2014. Disponível em: 
http:/faculdadedamas.edu.br/revistafd/index.php/arquitetura/index. Acesso em: 02 fev. 2020.

MIRANDA, Ewerton Bezerra Siqueira de; VECHIATO, Fernando Luiz. Wayfinding em ambientes informacionais: proposta de recomendações para avaliação no contexto da encontrabilidade da informação. Revista Informação na Sociedade Contemporânea, Natal, RN, v.1, n.3, jul/dez., 2017. Disponível em: https://periodicos.ufrn.br/informacao/article/view/10976. Acesso em: 02 fev. 2020.

MIRANDA, Májory Karoline Fernandes de Oliveira. Encontrabilidade e teoria da intencionalidade: propriedades para a informação. Pesquisa Brasileira em Ciência da Informação e Biblioteconomia, v. 14, n. 2, 2019.

MIRANDA, Májory Karoline Fernandes de Oliveira. $\mathbf{O}$ acesso à informação no paradigma pós-custodial: da aplicação da intencionalidade para findability. 2010. 353 f. Tese (Doutorado em Informação e Comunicação em Plataformas Digitais) - Faculdade de Letras, Universidade do Porto, Porto, 2010.

MORVILLE, Peter. Ambient Findability. Sebastopol: O'Reilly Media, 2005.

OLIVEIRA, Henry Poncio Cruz de. Arquitetura da Informação Pervasiva: contribuições conceituais. 2014. 203 f. Tese (Doutorado em Ciência da Informação) - Faculdade de Filosofia e Ciências, Universidade Estadual Paulista, Marília, 2014. Disponível em: http://www.marilia.unesp.br/Home/PosGraduacao/Cienciadalnformacao/Dissertacoes/oliveira_hpc_do_mar.pdf. Acesso em: 22 mar. 2020.

OLIVEIRA, Henry Poncio Cruz de; VIDOTTI, Silvana Aparecida Borsetti Gregório; BENTES PINTO, Virgínia. Arquitetura da Informação Pervasiva. São Paulo: Cultura Acadêmica, 2015.

RIBEIRO, Lúcia Gomes. Onde estou? Para onde vou? Ergonomia do ambiente construído: Wayfinding e Aeroportos. 2009. 120 f. Tese (Doutorado em Design) - Pontifícia Universidade Católica do Rio de Janeiro, Rio de Janeiro. Disponível em: https://www.maxwell.vrac.puc-

rio.br/colecao.php?strSecao=resultado\&nrSeq=32541@1. Acesso em: 02 fev. 2020.

ROSENFELD, Louis; MORVILLE, Peter.; ARANGO, Jorge. Information Architecture: for web and beyond. 4. ed. Sebastopol, CA: O'Reilly Media, 2015.

SMYTHE, Kelli Cristine Assis da Silva. Inclusão do usuário na fase inicial do processo de design para sistemas de wayfinding em ambientes

hospitalares já construídos. 2014. 182 f. Dissertação (Mestrado em Design) Setor de Artes, Comunicação e Design da Universidade Federal do Paraná, Curitiba, 2014. Disponível em: http://www.um.pro.br/prod/_pdf/001397.pdf. Acesso em: 20 jun. 2020. 
VECHIATO, Fernando Luiz. Encontrabilidade da Informação: contributo para uma conceituação no campo da Ciência da Informação. 2013. 206 f. Tese de Doutorado. Tese (Doutorado em Ciência da Informação) - Faculdade de Filosofia e Ciências, Universidade Estadual Paulista, Marília, 2013. Disponível em: https://repositorio.unesp.br/handle/11449/103365. Acesso em: 02 fev. 2020.

VECHIATO, Fernando Luiz; VIDOTTI, Silvana Aparecida Borsetti Gregório. Encontrabilidade da Informação: atributos e recomendações para ambientes informacionais digitais. Informação \& Tecnologia (ITEC), v. 1, n.2, p. 42-58, 2014. Disponível em: http://periodicos.ufpb.br/index.php/itec/article/view/22099. Acesso em: 02 fev. 2020.

\title{
CONTRUCTION FOR THE CONCEPT OF WAYFINDING IN INFORMATION SCIENCE
}

\begin{abstract}
Introduction: Wayfinding is associated, in the field of Urbanism, with the spatial orientation of subjects in urban spaces. However, its principles can be applied in informational environments, aiming to facilitate the finding of available information, which contributes significantly to the studies of Information Science. Objective: to present the foundations that define the concept of wayfinding in Information Science, according to the studies in Information Findability. Methodology: Based on a qualitative approach and through content analysis, articles are studied at national and international levels, found on the Portal de periódicos da Capes and the Base de Dados em Ciência da Informação, considering the period 2010 - 2020. The praxis was go through the content of these articles, understanding the categorizations and the core of meanings that underlie the concept of wayfinding in Information Science. Results: a concept of wayfinding converging with this moment of scientific history and culture is proposed. Conclusions: at the current historical and cultural scientific moment, research on wayfinding has an exponential potential in Information Science.
\end{abstract}

Descriptors: Wayfinding. Findability. Informational environments. Information and Technology.

\section{CONSTRUCCIÓN PARA EL CONCEPTO DE CAMINO EN CIENCIAS DE LA INFORMACIÓN}

\section{RESUMEN}

Introducción: Wayfinding se asocia, en el campo del urbanismo, con la orientación espacial de los sujetos en espacios urbanos. Sin embargo, sus principios se pueden aplicar en entornos informativos, con el objetivo de facilitar la búsqueda de información disponible, lo que contribuye significativamente a los estudios de las ciencias de la información. Objetivo: presentar los fundamentos que definen el concepto de orientación en Ciencias de la Información, de acuerdo con los estudios en Búsqueda de información. Metodología: Basado en un enfoque cualitativo y a través del análisis 
de contenido, los artículos se estudian a nivel nacional e internacional, se encuentran en el Portal de Capes Journals y en la Base de datos de ciencias de la información, considerando el período 2010-2020. La práctica fue revise el contenido de estos artículos, entendiendo las categorizaciones y el núcleo de los significados que subyacen al concepto de orientación en Ciencias de la Información. Resultados: se propone un concepto de orientación que converge con este momento de historia científica y cultura. Conclusiones: en el momento científico histórico y cultural actual, la investigación sobre la orientación tiene un potencial exponencial en Ciencias de la Información.

Descriptores: Wayfinding. Findability. Ambientes informativos. Información y tecnología.

Recebido em: 24.03 .2020

Aceito em: 04.08.2020 